\title{
Antitumor effects of a novel histone deacetylase inhibitor NK-HDAC-1 on breast cancer
}

\author{
ZHONG-HUA LI ${ }^{1,2}$, XIAO-BING ZHANG ${ }^{1}$, XI-QIAN HAN ${ }^{1}$, CONG-RAN FENG $^{2}$, \\ FENG-SHAN WANG $^{1}$, PENG GEORGE WANG ${ }^{2}$, JIE SHEN $^{2}$ and YI-KANG SHI ${ }^{1}$ \\ ${ }^{1}$ National Glycoengineering Research Center and School of Pharmaceutical Sciences, Shandong University, \\ Jinan, Shandong 250012; ${ }^{2}$ School of Pharmacy, Nankai University, Tianjin 300071, P.R. China
}

Received February 7, 2013; Accepted April 4, 2013

DOI: $10.3892 /$ or.2013.2434

\begin{abstract}
Histone deacetylases (HDACs) are overexpressed in various types of primary human cancer and have become attractive targets for cancer therapy. We designed and synthesized a series of new class of HDAC inhibitors (HDACi). Among these, S-(E)-3-(1-(1-(benzo[d]oxazol-2-yl)-2-methylpropyl)-1H-1,2,3triazol-4-yl)-N-hydroxyacrylamide (NK-HDAC-1) showed potent antitumor activity. In the present study, we examined the antitumor effects of NK-HDAC- 1 on breast cancer in vitro and in vivo. The inhibitory effects of NK-HDAC-1 on HDAC enzyme activity and cell growth were more potent compared to suberoylanilide hydroxamic acid (SAHA). NK-HDAC-1 caused G1 cell cycle arrest at concentrations below $0.2 \mu \mathrm{M}$ and $\mathrm{G} 2 / \mathrm{M}$ arrest at concentrations above $0.4 \mu \mathrm{M}$ through p21 upregulation and cyclin D1 downregulation. NK-HADC-1 induced hyperacetylation of histone $\mathrm{H} 3$ and $\mathrm{H} 4$ around the promoter region of p21. NK-HDAC-1 promoted apoptosis in MDA-MB-231 breast cancer cells by activating both the intrinsic and the extrinsic pathway NK-HDAC-1 at doses of 3, 10 and $30 \mathrm{mg} / \mathrm{kg}$ reduced the tumor volume in MDA-MB-231 xenografts by $25.9,48.8$ and $63.6 \%$, respectively. The results suggested that NK-HDAC-1 may be a promising therapeutic candidate in treating human breast cancer.
\end{abstract}

\section{Introduction}

Histone molecules are octamers composed of dimmers of the basic histone proteins $\mathrm{H} 2 \mathrm{~A}, \mathrm{H} 2 \mathrm{~B}, \mathrm{H} 3$ and $\mathrm{H} 4$. Acetylation and deacetylation of histones occur at lysine residues in the $\mathrm{N}$-terminal tail of all four histone proteins and are mediated by different families of histone acetylases (HATs) and histone deacetylases (HDACs). While the activity of HATs leads to a

Correspondence to: Dr Yi-Kang Shi, National Glycoengineering Research Center and School of Pharmaceutical Sciences, Shandong University, 44 West Wenhua Road, Jinan, Shandong 250012, P.R. China E-mail: shiyikang@sdu.edu.cn

Key words: histone deacetylase inhibitor, NK-HDAC-1, suberoylanilide hydroxamic acid, breast cancer, MDA-MB-231 cells hyperacetylated and 'open' chromatin conformation allowing transcriptional activity, HDAC activity represses transcriptional activity by condensing the chromatin package. The activity of HATs and HDACs is not restricted to histones, and a number of non-histone proteins have been identified as HAT/HDAC targets $(1,2)$. Currently, 18 HDACs are known; they are either zinc-dependent or nicotinamide adenine dinucleotide (NAD)-dependent and are generally divided into four classes, based on sequence homology to yeast counterparts. These HDAC substrates are directly or indirectly involved in numerous important cell pathways, including cell proliferation, differentiation, and apoptosis (3).

Overexpression of HDAC enzymes is observed in various primary human cancer tissues, which include the stomach, colon, breast, and prostate. Aberrant recruitment of HADCs correlates with the initiation and progression of various types of cancer $(4,5)$. As a result, HDACs have become attractive targets for cancer therapy and HDAC inhibitors (HDACi) have been discovered with different structural characteristics, including hydroximates, cyclic peptides, aliphatic acids and benzamides. Several HDACi have advanced into clinical trials $(6,7)$. A few of these compounds have shown potent antitumor activities in various types of hematologic and solid cancer. Currently, two HDACi - vorinostat (suberoylanilide hydroxamic acid; SAHA) and romidepsin (FK228; depsipeptide)- have been approved by the Food and Drug Administration for the treatment of patients with relapsed cutaneous T-cell lymphoma (7).

Several compounds in clinical developments seem to have limitations that include low oral bioavailability, poor in vivo stability, and undesirable safety profiles (1). Hence, there remains a significant clinical opportunity for efficacious HDACi that are safe and well tolerated. Previously, we designed and synthesized a series of new class of hydroxamate histone deacetylase inhibitors. Among them, S-(E)-3-(1-(1-(benzo[d] oxazol-2-yl)-2-methylpropyl)-1H-1,2,3-triazol-4-yl)-N-hydroxyacrylamide (NK-HDAC-1) (Fig. 1A) has more potent antitumor activity than SAHA $(8,9)$. In this study, we examined the functional effects of the novel HDACi NK-HDAC-1 on the activity of HADC enzyme and on the growth of breast cancer cells in vitro and in vivo. The possible molecular mechanisms underlying these effects were explored. These findings suggested that NK-HDAC-1 could be a potent HADC inhibitor against breast cancer. 


\section{Materials and methods}

Cell lines and cell culture. All human breast cancer cell lines used in this study were purchased from Shanghai Cell Bank, the Institute of Cell Biology, China Academy of Sciences (Shanghai, China). They were maintained in a humidified atmosphere containing $5 \% \mathrm{CO}_{2}$ at $37{ }^{\circ} \mathrm{C}$ in a different medium supplemented with $10 \% \mathrm{FBS}, 100 \mathrm{U} / \mathrm{ml}$ penicillin, and $100 \mu \mathrm{g} / \mathrm{ml}$ streptomycin. Breast cancer cell lines MDA-MB-231, MCF-7, SKBR3, MDA-MB-453, BT474 and T47D were cultured in RPMI-1640 or in DMEM medium. An immortalized human mammary epithelial cell line MCF10A was purchased from the American Type Culture Collection and cultured in DMEM medium supplemented with $5 \%$ horse serum, $20 \mathrm{ng} / \mathrm{ml}$ epidermal growth factor, $0.5 \mu \mathrm{g} / \mathrm{ml}$ hydrocortisone, $100 \mathrm{ng} / \mathrm{ml}$ cholera toxin, $10 \mu \mathrm{g} / \mathrm{ml}$ insulin, and penicillin/streptomycin.

Inhibition of cell growth in vitro. Cells were seeded in 96-well plates and incubated overnight. They were then treated for $72 \mathrm{~h}$ with varying concentrations of SAHA (Sigma-Aldrich, St. Louis, MO, USA) or NK-HDAC-1. Next, $20 \mu 1$ 3-(4,5-dimethylthiazol-2-yl)-2,5-diphenyl-tetrazolium bromide (MTT; Sigma-Aldrich) solution $(5 \mathrm{mg} / \mathrm{ml}$ in PBS) was added to each well and incubated for $4 \mathrm{~h}$ at $37^{\circ} \mathrm{C}$. After removing the medium, MTT formazan was dissolved in $150 \mu \mathrm{l}$ dimethyl sulfoxide (DMSO) and monitored by a microplate reader at a wavelength of $570 \mathrm{nM}$. The $\mathrm{IC}_{50}$ value was obtained using CalcuSyn software (Biosoft, Cambridge, UK).

HDAC enzyme activity assay in vitro. The deacetylase enzyme assay was based on a homogeneous fluorescence release assay. Purified HeLa cell nuclear extracts were incubated with NK-HDAC-1 diluted in various concentrations for $20 \mathrm{~min}$ in an assay buffer [ $25 \mathrm{mM}$ HEPES (pH 8.0), $137 \mathrm{mM}$ $\left.\mathrm{NaCl}, 1 \mathrm{mM} \mathrm{MgCl}_{2}, 2.7 \mathrm{mM} \mathrm{KCl}\right]$ at room temperature. The substrate Ac-Arg-Gly-Lys(Ac)-AMC was added to the reaction for further incubation at $37^{\circ} \mathrm{C}$. The reaction was discontinued by incubating it with trypsin at room temperature for $20 \mathrm{~min}$, allowing the release of fluorophore from the deacetylated substrate. The fluorescent signal was detected by a fluorometer at an excitation of $360 \mathrm{~nm}$, emission of $470 \mathrm{~nm}$, and cutoff at $435 \mathrm{~nm}$. HeLa cells were harvested by trypsinization, resuspended in two volumes of buffer $(60 \mathrm{mM}$ Tris- $\mathrm{HCl}, \mathrm{pH} 7.4$, $30 \%$ glycerol, $450 \mathrm{mM} \mathrm{NaCl}$ ), and lysed by three freeze and thaw cycles (dry ice and $30^{\circ} \mathrm{C}$ ). Cell debris was removed by centrifugation at 20,000 $\mathrm{xg}$ and the supernatant was aliquoted and stored at $-80^{\circ} \mathrm{C}$.

Cell cycle analysis by flow cytometry. Cells were trypsinized, fixed in ice-cold $70 \%$ ethanol, and then stored at $-20^{\circ} \mathrm{C}$. Prior to analysis, samples were washed twice in PBS and resuspended in a solution of propidium iodide $(50 \mathrm{mg} / \mathrm{ml})$ and RNase A $(0.5 \mathrm{mg} / \mathrm{ml})$ in PBS for $30 \mathrm{~min}$ in the dark. Data collected from 10,000 cells of each sample were analyzed by a flow cytometer (Becton-Dickinson Co., USA).

Annexin V-FITC apoptosis assay. Annexin V-FITC apoptosis assay was employed to determine viable, early apoptotic cells. According to the recommended protocols of the Annexin V-FITC kit (BD Pharmingen, USA), the cells were seeded at $4 \times 10^{5}$ cells $/ \mathrm{ml} /$ well in 6 -well plates. Following treatment with SAHA or NK-HDAC-1, MDA-MB-231 cells were harvested and washed twice with ice-cold phosphatebuffered saline (PBS) and resuspended in $100 \mu 1$ binding buffer. Next, $5 \mu 1$ Annexin V-FITC and $10 \mu 1$ propidium iodide were added and the mixture was incubated for $30 \mathrm{~min}$ in the dark. Finally, $400 \mu 1$ binding buffer was added to the cells and the mixture was analyzed using a flow cytometer (Becton-Dickinson Co.).

Western blotting. Cells were harvested after treatment and whole-cell lysate was prepared as described below. Cells were trypsinized, washed with PBS, and then lysed with a buffer containing $50 \mathrm{mM}$ Tris- $\mathrm{HCl}(\mathrm{pH} 7.5), 150 \mathrm{mM} \mathrm{NaCl}$, 2 mM EDTA, 2 mM EGTA, 1 mM dithiothreitol, $1 \%$ Nonidet P-40, $0.1 \%$ SDS, protease inhibitors (1 mM PMSF, $5 \mathrm{mg} / \mathrm{ml}$ aprotinin, $5 \mathrm{mg} / \mathrm{ml}$ leupeptin and $5 \mathrm{mg} / \mathrm{ml}$ pepstatin), and phosphatase inhibitors $(20 \mathrm{mM} \beta$-glycerophosphate, $50 \mathrm{mM}$ $\mathrm{NaF}$ and $1 \mathrm{mM} \mathrm{Na} \mathrm{VO}_{4}$ ). Lysates were incubated at $4^{\circ} \mathrm{C}$ for $20 \mathrm{~min}$ and centrifuged at $12,000 \mathrm{x}$ g for $15 \mathrm{~min}$. Equal amounts of lysate $(40 \mu \mathrm{g})$ were resolved by SDS-PAGE and transferred to the polyvinylidene difluoride membrane (Millipore, USA). Membranes were blocked in 5\% non-fat skim milk/TBST [20 mM Tris-HCl (pH 7.4), $150 \mathrm{mM} \mathrm{NaCl}$, and $0.1 \%$ Tween-20] at room temperature for $1 \mathrm{~h}$ and detected with primary antibodies at room temperature for $2 \mathrm{~h}$. The membranes were then blotted for $1 \mathrm{~h}$ at room temperature with an appropriate horseradish peroxidase-linked secondary antibody followed by enhanced chemiluminescence western blotting detection reagents (Amersham Pharmacia Biotech, USA). Cytosolic fractions were prepared by using mitochondria/cytosol fraction kit (BioVision). Primary antibody cyclin D1, p21, caspases-3, -9, -8, retinoblastoma (Rb), phosphorylated $\mathrm{Rb}$, actin, and secondary antibody were purchased from Santa Cruz Biotechnology, Inc. (Santa Cruz, CA, USA). Antiacetyl-histone $\mathrm{H} 3$ and $\mathrm{H} 4$ were purchased from Millipore. Poly(ADP-ribose) polymerase (PARP) and cytochrome $c$ were purchased from Cell Signaling Technology.

$R T$-PCR. Total RNA was extracted using TRIzol ${ }^{\circledR}$ reagent (Invitrogen). p21 mRNA expression was detected by RT-PCR according to the instructions (Qiagen). PCR primers for $\mathrm{p} 21$ were: 5'-GCCTGCCGCCGCCTCTTC-3' and 5'-GCCGCCT GCCTCCTCCCAACTC-3'. GAPDH was used as an internal control with forward primer, 5'-GGCAAATTCCATGG CACCGTCAAG-3' and reverse primer, 5'-GCAATGCCAG CCCCAGCGTCAAA-3'.

Chromatin immunoprecipitation. Analysis was performed by means of a chromatin immunoprecipitation assay kit protocol (Millipore). Soluble chromatin from MDA-MB-231 cells was immunoprecipitated with anti-acetylated histone $\mathrm{H} 3$ and $\mathrm{H} 4$ antibodies, respectively. PCR primer for the promoter region of the p21 gene was used to amplify the DNA isolated from the immunoprecipitated chromatin. p21 primers were 5'-CAGAGGAGGCGCCATGTCAG-3' and 5'-CCTGTGGGCGGATTAGGG-3'.

Acute toxicity study in BALB/c mice. BALB/c mice aged 7-8 weeks and weighing 20-22 $\mathrm{g}$ were purchased from the 
A<smiles>CC(C)[C@H](c1nc2ccccc2o1)n1cc(/C=C/C(=O)NO)nn1</smiles>

C

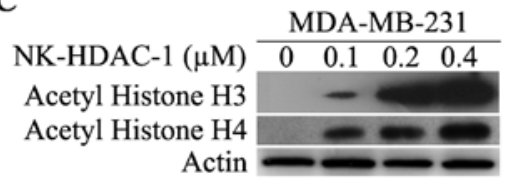

B

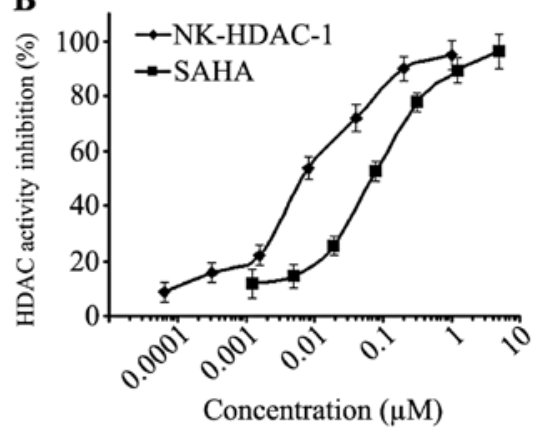

D

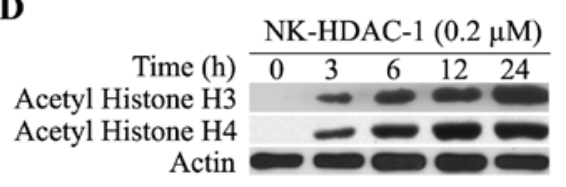

Figure 1. NK-HDAC-1 inhibits HDAC enzyme activity and causes acetylation of histone. (A) Structures of SAHA and NK-HDAC-1. (B) Inhibitory effects on HDAC enzyme activity. Total HDAC enzyme activity assay was performed as described in Materials and methods. (C) Effect of NK-HDAC-1 on acetylation of histone $\mathrm{H} 3$ and $\mathrm{H} 4$ in MDA-MB-231 cells. MDA-MB-231 cells were treated with either vehicle DMSO or the indicated concentrations of NK-HADC-1 for $24 \mathrm{~h}$. Whole-cell lysate was prepared and western blotting was performed. (D) MDA-MB-231 cells were treated with $0.2 \mu \mathrm{M}$ NK-HADC-1 for different times and were then harvested for western blotting.

Animal Center of Shandong University. All animal experiments were performed in compliance with the local Ethics Committee. Seventy mice were equally divided into seven groups of five males and five females each. NK-HDAC-1 was administered on the 1st day by intraperitoneal injection to groups of mice at doses of 300, 195, 126.8, 82.4, 53.6 and $34.8 \mathrm{mg} / \mathrm{kg}$ body weight at a ratio of $1 / 0.65$. The control group received $100 \mu \mathrm{l}$ DMSO only. The mortality and general conditions, i.e., energy, activity, hair, feces, behavior pattern, and other clinical signs, of all animals were observed continuously for 14 days after administration. The body weights of the mice were measured before dosing and on day 14 . All animals were sacrificed on the 14th day and the major organs of each mouse were examined after dissection.

Inhibition of tumor growth in vivo. The research protocol was in accordance with the institutional guidelines of the Animal Care and Use Committee at Shandong University. The animals were housed under pathogen-free conditions. Female BALB/c $(n u / n u)$ mice $(20 \pm 2 \mathrm{~g}, 4-6$ weeks old) were purchased from the Animal Center of the China Academy of Medical Sciences (Beijing, China). Breast cancer MDA-MB-231 cells of 5.0x10 suspended in $100 \mu 1 \mathrm{PBS}$ were subcutaneously inoculated into the lower right flank of the nude mice. The mice were divided randomly into four groups of six mice each when the tumors were $100-150 \mathrm{~mm}^{3}$ in size. The control group received solvent (DMSO) only while the positive control group received SAHA at $50 \mathrm{mg} / \mathrm{kg}$. The treatment groups received NK-HDAC-1 at the following doses: 100, 50, 30, 10 and $3 \mathrm{mg} / \mathrm{kg}$ body weight. The mice were injected intraperitoneally with $100 \mu \mathrm{l}$ DMSO, SAHA (diluted in $100 \mu 1$ DMSO), and NK-HDAC-1 (diluted in $100 \mu 1$ DMSO) on days 2, 3, 4, 6 and 7 of each week. All mice were then treated for 3 weeks. The diameter of the tumor was measured twice a week with a caliper. The tumor volume was calculated using the following formula: $\mathrm{v}=a b^{2} / 2$, where $a$ and $b$ are the long diameter and perpendicular short diameter of the tumor, respectively. At the end of the experiment, tumors were harvested and tumor weights were determined.

Statistical analysis. All quantitative data were subjected to ANOVA to determine the existence of significant differences between groups. For data groups satisfying the ANOVA criteria $(\mathrm{P}<0.05)$, individual comparisons were performed using the Student's t-test. $\mathrm{P} \leq 0.05$ was considered to indicate a statistically significant difference.

\section{Results}

NK-HDAC-1 inhibits total HDAC enzyme activity and increases acetylation of histone $H 3$ and $H 4$. NK-HDAC-1 was submitted to a deacetylation assay using a synthetic acetylated substrate to evaluate the inhibitory effect of NK-HDAC-1 on HDAC activity. As shown in Fig. 1B, NK-HDAC-1 significantly inhibited total HDAC activity in a dose-dependent manner with an $\mathrm{IC}_{50}$ value of $0.032 \pm 0.009 \mu \mathrm{M}$, which was much lower than that of SAHA $\left(\mathrm{IC}_{50}=0.218 \pm 0.051 \mu \mathrm{M}\right)$.

To confirm the ability of NK-HDAC-1 to inhibit deacetylation of HDACs in cells, western blotting was used to analyze acetylation of histone $\mathrm{H} 3$ and $\mathrm{H} 4$ in MDA-MB-231 breast cancer cells. NK-HDAC-1 dose-dependently increased the acetylation of histone $\mathrm{H} 3$ and histone $\mathrm{H} 4$ after the cells were treated for $24 \mathrm{~h}$ (Fig. 1C). Even $0.1 \mu \mathrm{M}$ NK-HDAC-1 markedly induced the acetylation of histone $\mathrm{H} 3$ and $\mathrm{H} 4$. The acetylation of histone $\mathrm{H} 3$ and $\mathrm{H} 4$ was detectable after MDA-MB-231 cells were treated with $0.5 \mu \mathrm{M}$ NK-HDAC- 1 for $3 \mathrm{~h}$ and they increased in a time-dependent manner for up to $24 \mathrm{~h}$ (Fig. 1D).

NK-HDAC-1 inhibits cell growth in breast cancer cells. NK-HDAC-1 was also entered into a screen at the National Cancer Institute (NCI60) where it was tested for growth inhibitory effects on a panel of cell lines representing various types of cancer. The results showed that NK-HDAC-1 was 


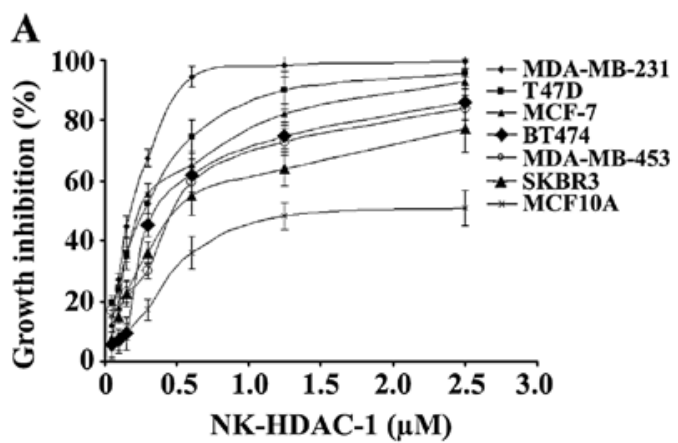

B IC IC $_{50}$ values of NK-HDAC-1 and SAHA

\begin{tabular}{lcc}
\hline & \multicolumn{2}{c}{$\mathrm{IC}_{50}(\mu \mathrm{M})$} \\
\cline { 2 - 3 } Cell lines & NK-HDAC-1 & SAHA \\
\hline MDA-MB-231 & $\mathbf{0 . 1 5} \pm 0.04$ & $\mathbf{2 . 2 5} \pm 0.09$ \\
T47D & $\mathbf{0 . 2 8} \pm 0.06$ & $3.26 \pm 0.12$ \\
MCF-7 & $\mathbf{0 . 3 5} \pm 0.08$ & $2.38 \pm 0.07$ \\
BT474 & $\mathbf{0 . 6 0} \pm 0.10$ & $\mathbf{2 . 4 7} \pm 0.08$ \\
MDA-MB-453 & $\mathbf{0 . 6 9} \pm 0.09$ & $\mathbf{1 . 9 0} \pm 0.33$ \\
SKBR3 & $\mathbf{0 . 9 5} \pm 0.11$ & $\mathbf{2 . 8 6} \pm 0.10$ \\
MCF10A & $\mathbf{1 . 6 1} \pm 0.06$ & $\mathbf{9 . 6 6} \pm 1.06$ \\
\hline
\end{tabular}

Figure 2. Growth inhibitory effects of NK-HDAC-1 on breast cancer cells. (A) Growth curves of breast cancer cells following treatment with NK-HDAC-1 for $72 \mathrm{~h}$. (B) $\mathrm{IC}_{50}$ values of NK-HDAC-1 and SAHA after breast cancer cells were treated for $72 \mathrm{~h}$. Cells were plated in 96 -well plates for $24 \mathrm{~h}$ and were then treated with various concentrations of NK-HDAC-1 or SAHA for $72 \mathrm{~h}$. Cell proliferation was determined with MTT assay. The $\mathrm{IC}_{50}$ value was obtained using CalcuSyn software. The results were derived from three independent experiments performed in triplicate.

approximately 1 order of magnitude more potent than SAHA against cancer cells growth (9). In order to test the effects of NK-HDAC-1 on the proliferation of breast cancer cells, we performed MTT assay in six human cancer cell lines as well as in an immortalized human breast epithelial cell MCF10A. After $72 \mathrm{~h}$ of treatment of the cells with NK-HDAC-1 and SAHA separately, NK-HDAC-1 markedly inhibited cell proliferation in all breast cancer cell lines in a dose-dependent manner (Fig. 2A), but it moderately affected the proliferation of MCF10A. The $\mathrm{IC}_{50}$ values of NK-HDAC-1 were significantly lower than those of SAHA on all cancer cells (Fig. 2B). MDA-MB-231 cells were most sensitive to NK-HDAC-1 among all the breast cancer cells and were selected for the subsequent experiments to explore the mechanisms by which NK-HDAC-1 inhibited breast cancer cell proliferation.

NK-HDAC-1 induces cell cycle arrest in MDA-MB-231 cells. HADCi generally inhibit the growth of tumor cells by causing them to arrest at a specific phase of the cell cycle. Cell cycle analysis was performed after $24 \mathrm{~h}$ of incubation with NK-HDAC-1 in order to detect the shifts in cell cycle distribution before a significant amount of cells underwent apoptosis. NK-HDAC-1 induced G1 arrest at concentrations below $0.20 \mu \mathrm{M}$ and $\mathrm{G} 2 / \mathrm{M}$ arrest at concentrations above $0.40 \mu \mathrm{M}$ after 24-h treatments in MDA-MB-231 cancer cells (Fig. 3A). As control, SAHA was found to cause G1 arrest at concentrations below $1.5 \mu \mathrm{M}$ and $\mathrm{G} 2 / \mathrm{M}$ arrest at concentrations above $3.0 \mu \mathrm{M}$, which were consistent with previous studies on the effects of SAHA on cell cycle distribution in breast cancer cells (10) (data not shown).

P21 is generally considered one of the targets of HDACi and an effector for HDACi-induced anticancer action. Following treatment with NK-HADC-1, p21 gene expression markedly increased and cyclin D1 expression markedly decreased. The phosphorylation levels of $\mathrm{Rb}$ decreased while $\mathrm{Rb}$ protein was unchanged after MDA-MB-231 cells were treated with NK-HADC-1 for $24 \mathrm{~h}$ (Fig. 3B). As measured by RT-PCR detection shown in Fig. 3C, p21 mRNA was induced by NK-HADC-1 in a dose-dependent manner in MDA-MB-231 cells.

To further elucidate whether NK-HADC-1 induced hyperacetylation of histone $\mathrm{H} 3$ and $\mathrm{H} 4$ around the promoter region of p21, chromatin immunoprecipitation was performed using primers corresponding to the region -597 to -342 bp upstream of the translation initiation site (ATG) of the p21 gene. Chromatin fragments from MDA-MB-231 cells cultured with or without NK-HADC-1 for $24 \mathrm{~h}$ were immunoprecipitated with antibody against acetylated histone $\mathrm{H} 3$ and $\mathrm{H} 4$, respectively. The chromatin fragments were then isolated and amplified using
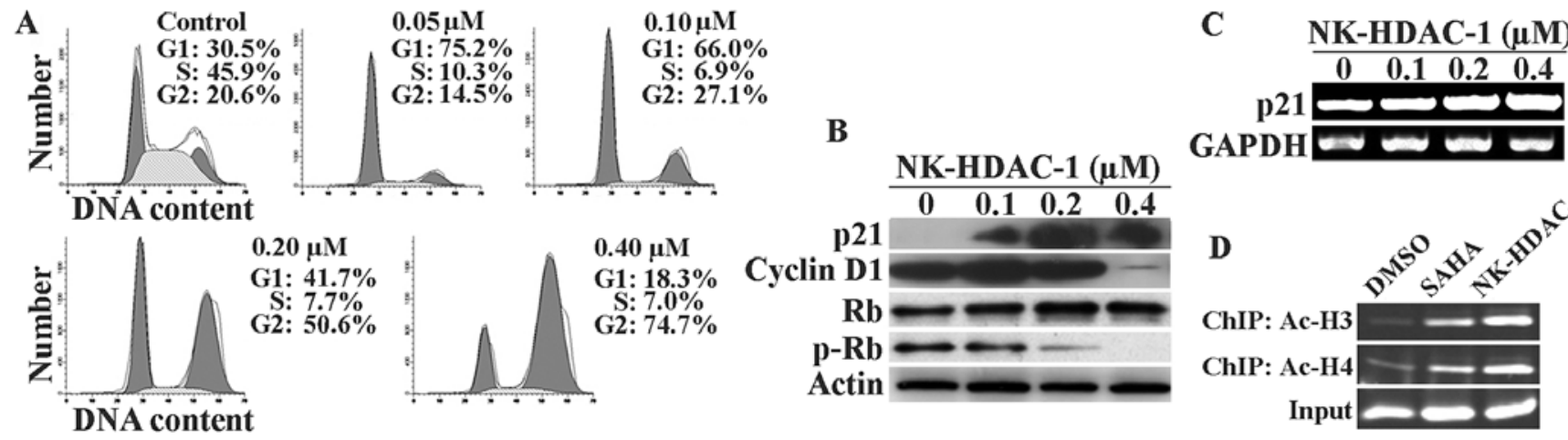

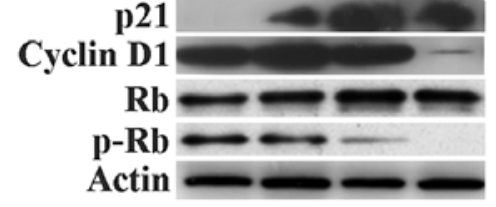

B

NK-HDAC-1 $(\mu \mathrm{M})$

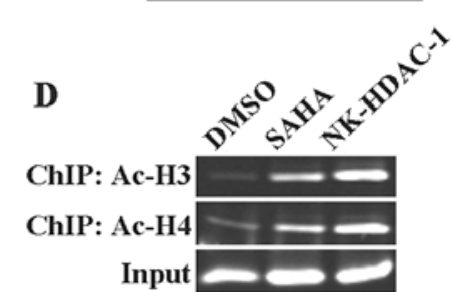

Figure 3. NK-HDAC-1 arrests breast cancer cell cycle progression. (A) Effects of NK-HDAC-1 on cell cycle distributions in viable MDA-MB-231 breast cancer cells. The cells were treated with NK-HDAC-1 for $24 \mathrm{~h}$ and then analyzed by flow cytometry. (B) Effect of NK-HDAC-1 on the protein expression of cell cycle regulators. MDA-MB-231 cells were treated with either vehicle DMSO or the indicated concentrations of NK-HADC-1 for 24 h. Whole-cell lysate was prepared and western blotting was performed. (C) p21 mRNA levels in MDA-MB-231 cells were examined by RT-PCR after cells were treated with NK-HADC-1 at the indicated concentrations for $24 \mathrm{~h}$. (D) ChIP assay. Chromatin fragments from MDA-MB-231 cells treated with $0.15 \mu \mathrm{M}$ NK-HADC-1 and $2.25 \mu \mathrm{M}$ SAHA for $24 \mathrm{~h}$ were immunoprecipitated with antibody against acetylated histone $\mathrm{H} 3$ and $\mathrm{H} 4$, respectively. The chromatin fragments were then isolated and amplified using PCR with p21 primers. Ac-H3 and Ac-H4 represent acetyl histone H3 and acetyl histone H4, respectively. Representative experiments were carried out at least three times. 
A
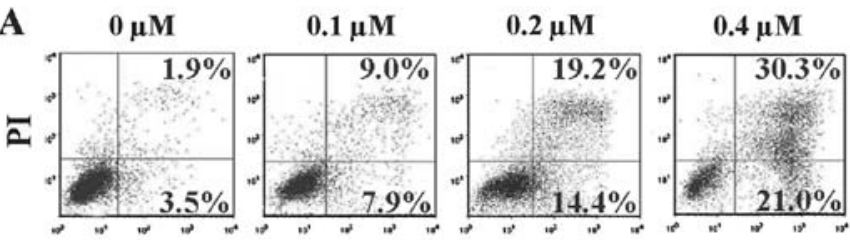

Annexin V

$\mathbf{B}$

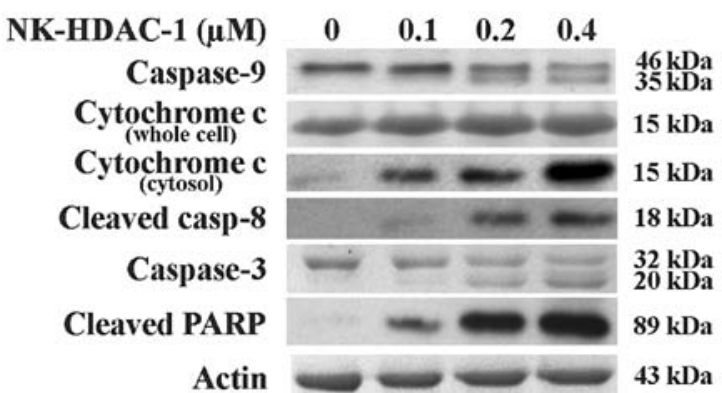

Figure 4. Effects of NK-HDAC-1 on apoptosis in MDA-MB-231 breast cancer cells. (A) MDA-MB-231 cells were treated with NK-HDAC-1 at the indicated concentrations for $48 \mathrm{~h}$ and were then analyzed for apoptosis by Annexin V-FITC assay. (B) Effects of NK-HDAC-1 on the expression levels of apoptosis-related proteins in MDA-MB-231 breast cancer cells. The cells were treated with NK-HDAC-1 for $48 \mathrm{~h}$ and western blotting was performed using whole-cell lysate to detect caspases- $9,-8,-3$, cytochrome $c$, PARP and actin. Cytosolic fraction was used to detect cytochrome $c$. Representative experiments were carried out at least three times.

PCR with p21 primers. Similar to SAHA, NK-HADC-1 induced hyperacetylation around the promoter region of $\mathrm{p} 21$ in MDA-MB-231 cells (Fig. 3D). This result strongly suggested that p21 upregulation is mainly due to enhancement of acetylation of histones $\mathrm{H} 3$ and $\mathrm{H} 4$ around the promoter region of $\mathrm{p} 21$.

NK-HDAC-1 induces apoptosis in MDA-MB-231 cells by activating both the intrinsic and the extrinsic pathway. Apoptosis is believed to be the common cause of cell killing by chemotherapy treatment as well as by HDACi. The rate of apoptotic cells was then detected through Annexin V assay. NK-HDAC-1 induced apoptosis in a dose-dependent manner in MDA-MB-231 cells (Fig. 4A). Early apoptotic cells increased from 7.9 to $21.0 \%$ and late apoptotic cells increased from 9.0 to $30.3 \%$ following NK-HDAC-1 treatment of 0.1 to $0.4 \mu \mathrm{M}$ for $48 \mathrm{~h}$. These drug concentrations were selected as they were near the $\mathrm{IC}_{50}$ concentration of NK-HDAC-1 on MDA-MB-231 cells. Western blot analysis showed that the release of cytochrome $c$ from mitochondria to the cytosol was dose-dependently increased while cytochrome $c$ in whole cells was unchanged (Fig. 4B). Both caspase- 9 and -8 activation was also confirmed by the appearance of cleaved caspase- 9 and cleaved caspase- 8 . The bands of cleaved caspase- 3 and cleaved PARP, as a result of apoptosis, were clearly observed (Fig. 4B). Collectively, these results suggested that the major mechanism of apoptosis induced by NK-HDAC-1 was activation of the intrinsic and the extrinsic pathway.

Acute toxicity of NK-HDAC-1 in BALB/c mice. To investigate the maximal tolerance dose of NK-HDAC-1 in vivo, acute toxicity analysis in $\mathrm{BALB} / \mathrm{c}$ mice was performed. NK-HDAC-1 was injected intraperitoneally into six groups of ten mice
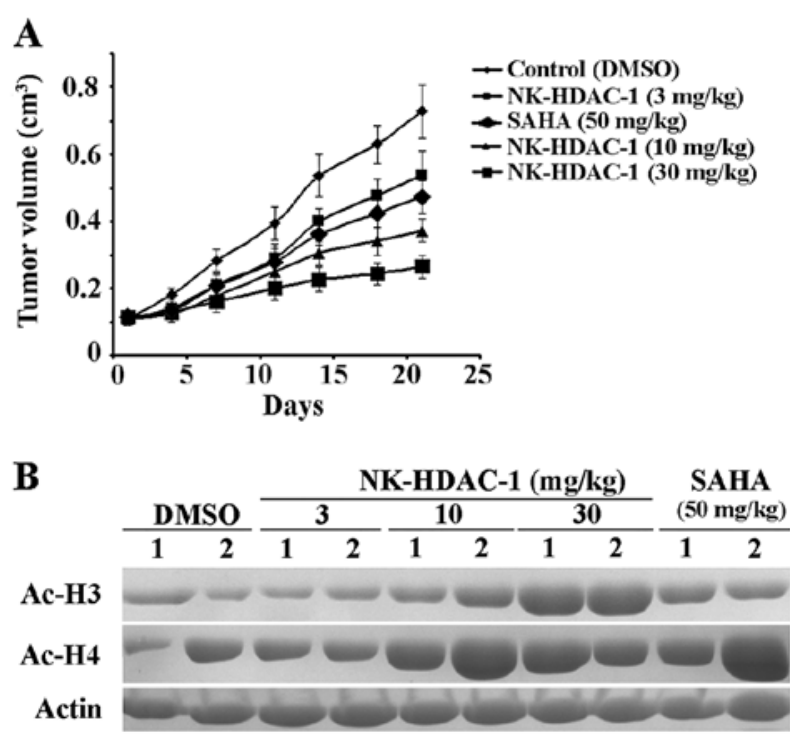

Figure 5. Antitumor effects of NK-HDAC-1 on nude mice bearing breast cancer xenograft. The nude mice bearing breast cancer MDA-MB-231 cells were treated as described in Materials and methods. (A) Tumor size growth curves were determined by calculating the volume size of individual tumors. (B) NK-HDAC-1 increased acetylation of histone $\mathrm{H} 3$ and $\mathrm{H} 4$ in tumors. Two tumors from each group were selected to determine the acetylation of histone $\mathrm{H} 3$ and $\mathrm{H} 4$ by western blotting. Ac- $\mathrm{H} 3$ and Ac-H4 represent acetyl histone $\mathrm{H} 3$ and acetyl histone $\mathrm{H} 4$, respectively.

at doses of $300,195,126.8,82.4,53.6$ and $34.8 \mathrm{mg} / \mathrm{kg}$ body weight. The control group was injected with solvent DMSO. Following exposure, mortality and changes in the body weight of the mice were recorded for 14 days. All ten mice treated with $300 \mathrm{mg} / \mathrm{kg}$ died within 2 days. Nine of the ten mice treated with $195 \mathrm{mg} / \mathrm{kg}$ died within 2 days. Two mice in the group treated with $126.8 \mathrm{mg} / \mathrm{kg}$ and one mouse in the group treated with $82.4 \mathrm{mg} / \mathrm{kg}$ died. All deaths occurred in the first two days and no mice died during the last 12 days of the observation period. No mice died in the groups treated with NK-HDAC-1 at doses of 53.6 and $34.8 \mathrm{mg} / \mathrm{kg}$. None of the surviving mice showed any abnormal signs and the body weights of the mice were significantly unchanged during the period of the test (data not shown). The results indicated that the maximal tolerance dose of NK-HDAC-1 in BALB/c mice was $>53.6 \mathrm{mg} / \mathrm{kg}$.

NK-HDAC-1 inhibits breast tumor growth. To further assess the antitumor effects of NK-HDAC-1 in vivo, athymic mice bearing MDA-MB-231 xenograft were treated 5 days a week for 21 days with vehicle or SAHA at $50 \mathrm{mg} / \mathrm{kg}$ or with NK-HDAC-1 at doses of $100,50,30,10$ and $3 \mathrm{mg} / \mathrm{kg}(\mathrm{n}=6)$. Four of the six mice in the group treated with $100 \mathrm{mg} / \mathrm{kg}$ NK-HDAC-1 died at the end of the experiment. One of the six mice in the group treated with $50 \mathrm{mg} / \mathrm{kg}$ NK-HDAC-1 died and the mean body weight of the five surviving mice decreased significantly from $19.8 \mathrm{~g}$ on day 1 to $16.8 \mathrm{~g}$ on day 21 . Compared with the control group, NK-HDAC-1 at doses of 30,10 and $3 \mathrm{mg} / \mathrm{kg}$ did not cause overt signs of toxicity or significant weight loss (data not shown). Tumor volumes in MDA-MB-231 xenografts decreased by $63.6,48.8$ and $25.9 \%$, respectively (Fig. 5A). In the same experiment, $50 \mathrm{mg} / \mathrm{kg}$ SAHA, a positive control, inhibited tumor growth by $35.0 \%$. 
The expression of acetylation of both histone $\mathrm{H} 3$ and $\mathrm{H} 4$ in tumor was determined by western blot analysis to investigate whether NK-HDAC-1 affects HDAC enzyme activity in vivo. Two tumors from each group were selected for this analysis. As shown in Fig. 5B, NK-HDAC-1 enhanced the acetylation of histone $\mathrm{H} 3$ and $\mathrm{H} 4$ in tumor sample, thus confirming NK-HDAC-1 inhibited HDAC activity in vivo.

\section{Discussion}

Since HDACs regulate a variety of cell functions that are involved in cell survival, cell cycle progression, angiogenesis, and immunity, they are considered promising targets for cancer therapy. In the current study, we provided the antitumor effect of a novel hydroxamate histone deacetylase inhibitor, NK-HDAC-1, in breast cancer in vitro and in vivo.

NK-HDAC-1 exhibited more potent antiproliferative effects than SAHA. In contrast to SAHA with $\mathrm{IC}_{50}$ at $0.218 \pm 0.051 \mu \mathrm{M}$, NK-HDAC-1 had higher inhibitory effects on total HDAC enzyme activity with $\mathrm{IC}_{50}$ at $0.032 \pm 0.009 \mu \mathrm{M}$. The expression of acetylated histone $\mathrm{H} 3$ and $\mathrm{H} 4$ was markedly enhanced by NK-HDAC-1 even at very low concentrations.

NK-HDAC-1 showed a potent growth inhibitory effect against breast cancer cells in a dose-dependent manner. Moreover, it was quite resistant to the immortalized human epithelial breast cell line MCF10A, which was consistent with previous findings that normal cells appear to be relatively resistant to HDACi $(11,12)$.

Increasing evidence has revealed that HDACi suppress cancer cell growth by inducing cell cycle arrest at the G1 and/or G2/M phase $(13,14)$. SAHA caused G1 and/or G2/M arrest in various cancer cells $(15,16)$. Similarly, NK-HDAC-1 induced G1 arrest at lower concentrations and G2/M arrest at higher concentrations.

Several studies demonstrated that cyclin-dependent kinases (CDKs) inhibitor p21 and cyclin D1 are important regulators of the cell cycle in breast cancer. p21 inhibits CDK2cyclin $\mathrm{E}$, with the consequent inhibition of CDK2-dependent phosphorylation of $\mathrm{Rb}$, thus inhibiting E2F1-dependent gene transcription and progression into and through the $\mathrm{S}$ phase. p21 also inhibits the kinase activity of CDK2-cyclin A and CDK1-cyclin A, which are required for progression through the $\mathrm{S}$ phase and into $\mathrm{G} 2$ respectively. Additionally, p21 inhibits the kinase activity of CDK1-cyclin B1, thus inhibiting progression through $\mathrm{G} 2$ and $\mathrm{G} 2 / \mathrm{M}$ (17). The primary function of cyclin D is to activate the CDKs CDK4 and CDK6 as a regulatory partner and then $\mathrm{CDK}$ s promote $\mathrm{Rb}$ phosphorylation and activation of E2F-responsive gene (18). In this study, NK-HDAC-1 markedly increased both p21 protein expression and mRNA level. Cyclin D1 and the phosphorylation of $\mathrm{Rb}$ were dose-dependently decreased while $\mathrm{Rb}$ protein level was unaltered. In this study, the increase of p21 and the decrease of cyclin D1 may act together to account for the reduced CDK activity, causing dephosphorylation of Rb, which blocks E2F activities in the transcription of genes for G1 progression and G1/S transition. This study revealed that NK-HDAC-1 differentially regulated cell cycle progression depending on its concentration, and that its inhibition of cancer cell growth may be mediated, at least in part, by blocking cell cycle progression.
HDACi can affect transcription by inducing acetylation of histones, transcription factors and other proteins regulating transcription (19). In this report, we identified that NK-HDAC-1 induced hyperacetylation of histone $\mathrm{H} 3$ and $\mathrm{H} 4$ around the promoter region of $\mathrm{p} 21$, which may enhance $\mathrm{p} 21$ mRNA level and protein expression.

All HDACi have been reported to activate either an extrinsic or intrinsic pathway or both of these cell death pathways in several cancer models $(20,21)$. The death-receptor pathway is activated when ligands, such as Fas or TRAIL, bind to their death receptors, causing the activation of caspase- 8 . The mitochondrial pathway is activated by stress stimuli (chemotherapeutic agents) that disrupt the mitochondrial membrane, causing the release of cytochrome $c$. Cytochrome $c$ release leads to apoptosome formation and activation of caspase-9. Caspases- 8 and -9 can then cleave caspases-3, -6, and -7, culminating in apoptosis (22). Annexin V assay in this study showed that NK-HDAC-1 promoted apoptosis in a dose-dependent manner. Western blotting showed that cytochrome $c$ translated from mitochondria to cytosol with activation of caspase-9. The activation of caspases- 9 and -8 resulted in enhancement of cleaved caspase- 3 and PARP. These results suggested that NK-HDAC-1 induced apoptosis via both the intrinsic and the extrinsic pathway and that the induction of apoptosis may be another mechanism responsible for growth inhibition by NK-HDAC-1.

The acute toxicity experiment indicated that the maximal tolerance dose of NK-HDAC-1 in BALB/c mice was higher than $53.6 \mathrm{mg} / \mathrm{kg}$. In the nude mice xenograft model treated five times a week for three weeks, one in six mice died in the group treated with $50 \mathrm{mg} / \mathrm{kg}$ NK-HDAC-1. However, treatment with NK-HDAC-1 at doses of 30, 10 and $3 \mathrm{mg} / \mathrm{kg}$ inhibited tumor growth without any signs of toxicity by $63.6,48.8$ and $25.9 \%$, respectively. These results showed that NK-HDAC-1 had potent antitumor activity in vivo.

In conclusion, results of the present study demonstrated that the novel hydroxamate HDAC inhibitor NK-HDAC-1 inhibited the growth of cancer cells in vitro and in vivo and that the growth inhibitory effect of NK-HDAC-1 may be mediated by cell cycle arrest and apoptosis. The results suggest that NK-HDAC-1 is a potential therapeutic candidate for cancer therapy.

\section{Acknowledgements}

This study was supported by grants from the National Natural Science Foundation of China (no. 21072105) and the Independent Innovation Foundation of Shandong University (no. 2009DX002).

\section{References}

1. Glaser KB: HDAC inhibitors: clinical update and mechanismbased potential. Biochem Pharmacol 74: 659-671, 2007.

2. Zhou Q, Chaerkady R, Shaw PG, Kensler TW, Pandey A and Davidson NE: Screening for therapeutic targets of vorinostat by SILAC-based proteomic analysis in human breast cancer cells. Proteomics 10: 1029-1039, 2010.

3. Struhl K: Histone acetylation and transcriptional regulatory mechanisms. Genes Dev 12: 599-606, 1998.

4. Marks PA and Xu WS: Histone deacetylase inhibitors: potential in cancer therapy. J Cell Biochem 107: 600-608, 2009. 
5. Hagelkruys A, Sawicka A, Rennmayr M and Seiser C: The biology of HDAC in cancer: the nuclear and epigenetic components. Handb Exp Pharmacol 206: 13-37, 2011.

6. Ma X, Ezzeldin HH and Diasio RB: Histone deacetylase inhibitors: current status and overview of recent clinical trials. Drugs 69: 1911-1934, 2009.

7. Gryder BE, Sodji QH and Oyelere AK: Targeted cancer therapy: giving histone deacetylase inhibitors all they need to succeed. Future Med Chem 4: 505-524, 2012.

8. Hou J, Feng C, Li Z, et al: Structure-based optimization of click-based histone deacetylase inhibitors. Eur J Med Chem 46: 3190-3200, 2011

9. Hou J, Li Z, Fang Q, et al: Discovery and extensive in vitro evaluations of NK-HDAC-1: a chiral histone deacetylase inhibitor as a promising lead. J Med Chem 55: 3066-3075, 2012.

10. Huang L and Pardee AB: Suberoylanilide hydroxamic acid as a potential therapeutic agent for human breast cancer treatment. Mol Med 6: 849-866, 2000.

11. Armeanu S, Pathil A, Venturelli S, Mascagni P, et al: Apoptosis on hepatoma cells but not on primary hepatocytes by histone deacetylase inhibitors valproate and ITF2357. J Hepatol 42: 210-217, 2005

12. Garber K: HDAC inhibitors overcome first hurdle. Nat Biotechnol 25: 17-19, 2007.

13. Marks PA and Dokmanovic M: Histone deacetylase inhibitors: discovery and development as anticancer agents. Expert Opin Investig Drugs 14: 1497-1511, 2005.

14. Ungerstedt JS, Sowa Y, Xu WS, et al: Role of thioredoxin in the response of normal and transformed cells to histone deacetylase inhibitors. Proc Natl Acad Sci USA 102: 673-678, 2005.
15. Kumagai T, Wakimoto N, Yin D, et al: Histone deacetylase inhibitor, suberoylanilide hydroxamic acid (Vorinostat, SAHA) profoundly inhibits the growth of human pancreatic cancer cells. Int J Cancer 121: 656-665, 2007.

16. Yin D, Ong JM, Hu J, et al: Suberoylanilide hydroxamic acid a histone deacetylase inhibitor: effects on gene expression and growth of glioma cells in vitro and in vivo. Clin Cancer Res 13: 1045-1052, 2007.

17. Gartel AL: p21(WAF1/CIP1) and cancer: a shifting paradigm? Biofactors 35: 161-164, 2009.

18. Kim JK and Diehl JA: Nuclear cyclin D1: an oncogenic driver in human cancer. J Cell Physiol 220: 292-296, 2009.

19. Gui CY, Ngo L, Xu WS, Richon VM and Marks PA: Histone deacetylase (HDAC) inhibitor activation of $\mathrm{p} 21^{\mathrm{WAF} 1}$ involves changes in promoter-associated proteins, including HDAC1. Proc Natl Acad Sci USA 101: 1241-1246, 2004.

20. Huang L, Sowa Y, Sakai T and Pardee AB: Activation of the p21 $1^{\text {WAF1/CIP1 }}$ promoter independent of p53 by the histone deacetylase inhibitor suberoylanilide hydroxamic acid (SAHA) through the Sp1 sites. Oncogene 19: 5712-5719, 2000.

21. Rikiishi H: Autophagic and apoptotic effects of HDAC inhibitors on cancer cells. J Biomed Biotechnol 2011: 830260, 2011.

22. Emanuele S, Lauricella M and Tesoriere G: Histone deacetylase inhibitors: apoptotic effects and clinical implications (Review). Int J Oncol 33: 637-646, 2008. 